\title{
An Experimental Model for Inducing Periodontal Pathology in Rat: Histopathological and Enzymatic Aspects
}

Adina Bianca BOȘCA ${ }^{1}$, Aranka ILEA ${ }^{1}$, Alina Simona ȘOVREA ${ }^{1}$, Anne Marie CONSTANTIN ${ }^{1}$, Flavia RUXANDA $^{2}$, Vasile RUS ${ }^{2 *}$, Cristian RAȚIU ${ }^{3}$, Viorel MICLĂUŞ ${ }^{2}$

1 "Iuliu Haţieganu" University of Medicine and Pharmacy, Cluj-Napoca, Romania

${ }^{2}$ University of Agricultural Sciences and Veterinarian Medicine, Cluj-Napoca, Romania

${ }^{3}$ University of Oradea, Faculty of Medicine and Pharmacy, Oradea, Romania

*Corresponding author e-mail: vasilerus2002@yahoo.com

Bulletin UASVM Veterinary Medicine 71(2) / 2014,

Print ISSN 1843-5270; Electronic ISSN 1843-5378

DOI:10.15835/buasvmcn-vm: 10536

\begin{abstract}
In our study, we induced periodontal pathology in rats by repetitive acute lesions to the gingival epithelium. In order to assess the evolution of the periodontal pathology, we recorded the clinical and histopathological aspects of the gingiva, and Matrix metalloproteinase-8 (MMP-8) levels in serum and saliva at baseline and during the inflammation progression. According to our findings, this experimental model was consistent with the periodontal disease in humans and could be used to test the efficiency and safety of novel therapies prior to their application in patients.
\end{abstract}

Keywords: experimental model, MMP-8, periodontal pathology, rat.

Introduction. Experimental models represent an essential link between hypotheses and human patients (Graves et al., 2012). Even though animal models provide useful information, it is controversial if these data can be translated to humans (Oz and Puleo, 2011); this is the case of periodontal disease, a typical human pathology, in which the complex mechanisms involved in the initiation and progression of the periodontal inflammation cannot be identically reproduced in animals (Graves et al., 2012). For this reason, up to date, there are no perfect animal models for reproducing periodontal disease. Matrix metalloproteinase-8 (MMP-8), is considered as a marker of periodontal inflammation (Gursoy et al., 2010; Gupta et al., 2014).

Aims and objectives. The aim of the present study was to test a new protocol for inducing the periodontal pathology in rat.

Materials and methods. Periodontal pathology was induced in 10 female Wistar rats by repetitive acute gingival lesions in relation to the lower incisors. The interventions were performed daily, from D1 to D16; a periodontal curette was forced into the sulcus, the circular periodontal ligament was sectioned, and the gingival epithelium was detached from the teeth. The evolution of the lesions was monitore d by clinical, histopathological and enzymatic examinations. Clinically, we recorded the degree of gingival inflammation by assessing the colour (erythema/cyanosis), the consistency (firm/soft), the increased volume (oedema), and bleeding (which occurred upon probing the gingival sulcus with a periodontal probe, or spontaneously). Histopathologically, on D1, D3, D6, D8, D16 we assessed the degenerative changes in the gingival epithelium and chorion, the microcirculatory alterations and the inflammatory infiltrate composition. MMP-8 salivary and serum levels were measured using ELISA (Enzymelinked Immunosorbent Assay) technique (Kit for Rat Matrix Metalloproteinase 8, provided by Uscn Life Science Inc. Wuhan). 

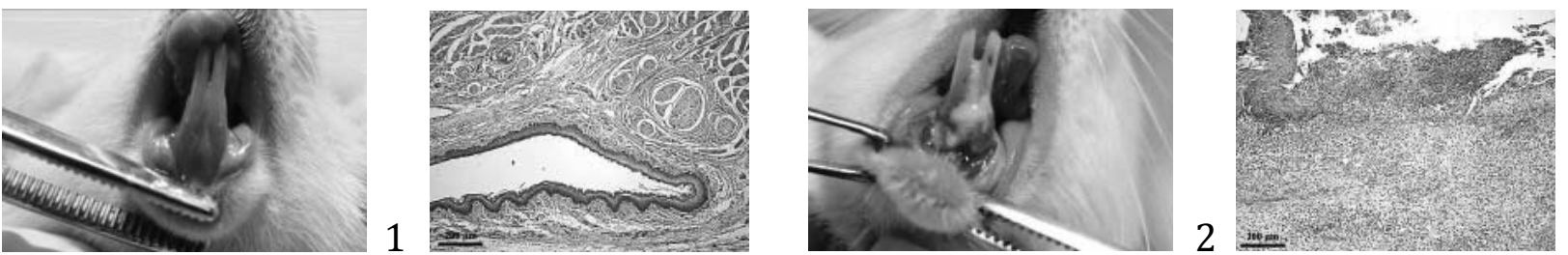

Fig. 1 - 2. Clinical (the left image) and histological (the right image) aspects of the gingiva (Goldner's trichromic stain); 1- aspects of the healthy gingiva on D1; 2 - aspects of the advanced periodontal disease on

D16.
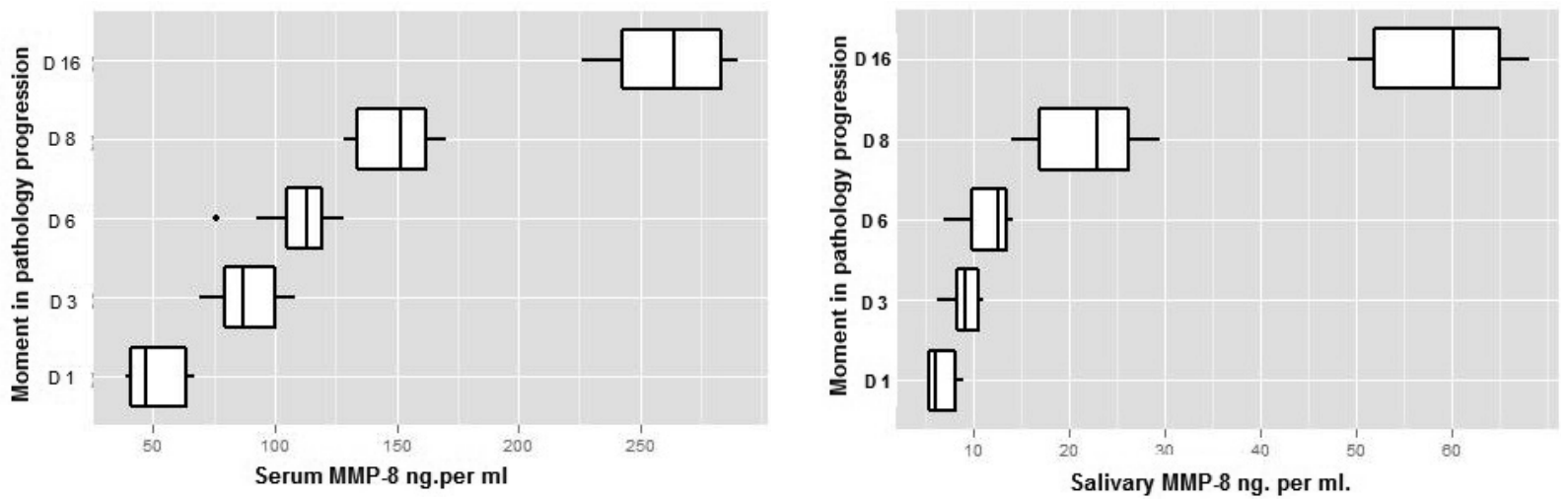

Fig. 3. Variations in MMP-8 serum and salivary levels during periodontal inflammation progression.

Results and discussions. The clinical and histopathological examinations have recorded, stage by stage, the structural changes throughout the initiation and progression of the disease. On D1, the normal aspect of the gingiva was recorded (Fig.1); on D16, the rats displayed clinical and histopathological features of advanced periodontal disease, consisting in: severe inflammation and spontaneous bleeding, perivascular and diffuse oedemas, hemorrhagic and necrotic lesions and extended infiltration with lymphocytes and granulocytes (Fig.2).

Salivary and serum MMP-8 levels showed a continuous and progressive increase, consistent with the degree of gingival inflammation (Fig. 3).

Our findings were similar to experimental studies using different methodology (Yang et al., 2013). Moreover, the recorded clinical, histopathological and enzymatic changes were consistent, in evolution, to the specific aspects reported in human chronic periodontitis (Gursoy et al., 2010; Gupta et al., 2014).
Conclusion. The animal model tested in our study proved to be functional and could be used for demonstrating the cause-effect relationship and for testing innovative therapies in periodontal disease, prior to their implementation in humans.

\section{References}

1. Graves DT, Kang J, Andriankaja O, Wada K, Rossa C Jr. (2012). Animal models to study host-bacteria interactions involved in periodontitis. Front Oral Biol. 15:117-32.

2. Oz HS, Puleo DA. (2011). Animal models for periodontal disease. J Biomed Biotechnol. 754857.

3. Gursoy UK, Könönen E, Pradhan-Palikhe P, Tervahartiala T, Pussinen PJ, Suominen-Taipale L, Sorsa T. (2010). Salivary MMP-8, TIMP-1, and ICTP as markers of advanced periodontitis. J Clin Periodontol. 37(6):487-93.

4. Gupta N, Gupta ND, Gupta A, Khan S, Bansal N. (2014). Role of salivary matrix metalloproteinase-8 (MMP-8) in chronic periodontitis diagnosis. Front Med. Aug 6.

5. Yang D, Wang J, Ni J, Shang S, Liu L, Xiang J, Li C. (2013). Temporal expression of metalloproteinase-8 and -13 and their relationships with extracellular matrix metalloproteinase inducer in the development of ligatureinduced periodontitis in rats. J Periodontal Res.48(4):411-9. 